\title{
Furostanol Saponins from Asparagus cochinchinensis and Their Cytotoxicity
}

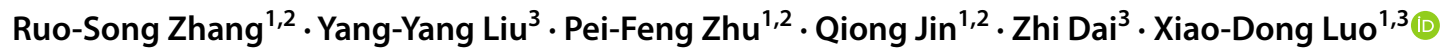

Received: 16 September 2021 / Accepted: 25 October 2021 / Published online: 5 November 2021

(c) The Author(s) 2021

\begin{abstract}
Phytochemical investigation on the roots of Asparagus cochinchinensis led to the isolation of one new furostanol saponin, named 26- $O$ - $\beta$-D-glucopyranosyl-22 $\alpha$-hydroxyl- $(25 R)-\Delta^{5(6)}$-furost-3 $\beta, 26$-diol-3- $O$ - $\alpha$-L-rhamnopyranosyl- $(1 \rightarrow 2)$ - $[\beta$-Dglucopyranosyl-( $1 \rightarrow 4)-\alpha$-L-rhamnopyranosyl- $(1 \rightarrow 4)]-\beta$-D-glucopyranoside $(\mathbf{1})$, along with three known congeners $(\mathbf{2}-\mathbf{4})$. The structure of new saponin was elucidated via comprehensive inspection of its HRMS and NMR spectral data as well as chemical technology, whereas those of known ones were identified by comparison of their NMR and MS spectral data with those reported in literatures. All isolated saponins were evaluated for their cytotoxic effects on two human liver (MHCC97H) and lung adenocarcinoma (H1299) cancer cells in vitro. Among them, both $\mathbf{1}$ and $\mathbf{2}$ showed significant cytotoxicity against above mentioned cell lines. Further studies revealed that these two saponins could significantly inhibit their proliferation of MHCC 97H and H1299 cells.
\end{abstract}

\section{Graphic Abstract}
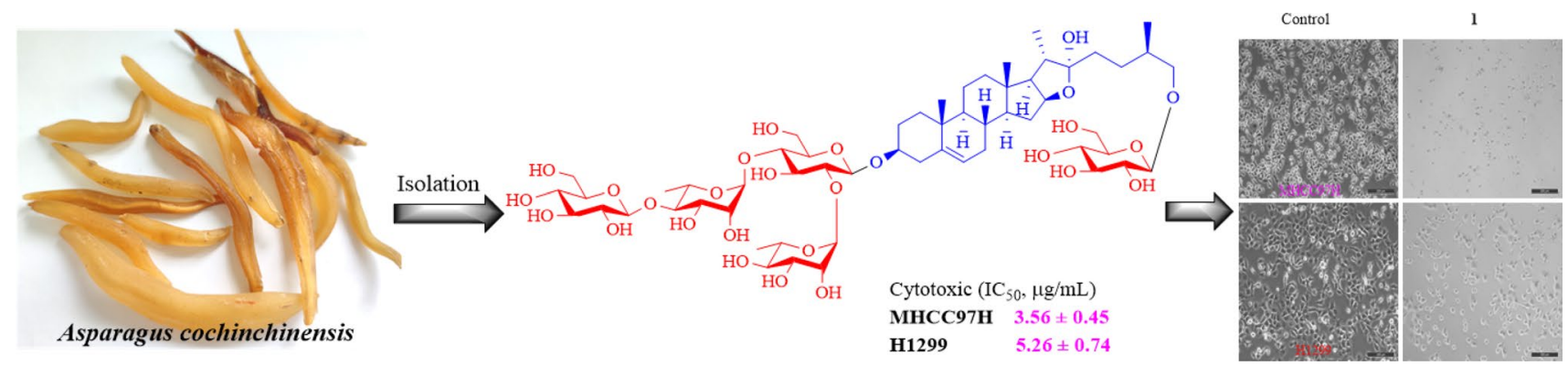

Keywords Steroid saponins $\cdot$ Asparagus cochinchinensis $\cdot$ Cytotoxicity $\cdot$ Structural elucidation

Xiao-Dong Luo

xdluo@mail.kib.ac.cn

1 State Key Laboratory of Phytochemistry and Plant Resources in West China and Yunnan Key Laboratory of Natural Medicinal Chemistry, Kunming Institute of Botany, Chinese Academy of Sciences, Kunming 650201, China

2 University of Chinese Academy of Sciences, Beijing 100049, China

3 Key Laboratory of Medicinal Chemistry for Natural Resource, Ministry of Education, Yunnan Provincial Center for Research \& Development of Natural Products, School of Chemical Science and Technology, Yunnan University, Kunming, China

\section{Introduction}

Steroid saponins, whose aglycones were usually a spirostanol or its derivatives [1], were commonly found from roots, tubers, leaves, blooms or seeds in more than 100 families of plants $[2,3]$. Compared with other glycosides, the strong foam-forming property in aqueous solution of steroidal saponins was their main feature $[2,4]$. Previous researches revealed steroidal saponins possessed various pharmacological activities, such as antifungal [5], hypocholesterolemic [6], antimitotic [7] and cAMP phosphodiesterase inhibitory [8] effects. Among them, a large number of publications have revealed steroid saponins shared different 
cytotoxic properties that promoted their potential as anticancer drugs or adjuvants $[9,10]$.

Asparagus cochinchinensis, belonging to the genus Asparagus (Liliaceae), is well-known as "Tianmendong" in China. Its roots have been historically used in Chinese folk medicine for the treatment of cough, acute and chronic bronchitis, chronic pharyngitis, hemorrhoids, and tumors for thousands of years [11]. Apart from steroidal saponins [12], phenolic compounds [13], norlignans [14] and alkaloids [15] have been isolated from this plant as revealed by previous phytochemical studies. However, steroidal saponins obtained from title species were proved to be its major and bioactive components responsible for its cytotoxic [16], antiinflammatory [17], hepatotoxic and nephrotoxic [18], and anti-neuroinflammatory [11] properties. In continuation of a search for bioactive constituents from plants of the Yunnan province [19], a chemical investigation was performed on the roots of $A$. cochinchinens. As a result, a total of steroidal saponins (1-4) were isolated and identified including one new and three previously described furostan-type steroidal saponins. Their cytotoxic effects on two human cancer cells MHCC97H and H1299 were also evaluated (Fig. 1).

\section{Results and Discussion}

Saponin 1 was obtained as a white amorphous powder. It had a molecular formula of $\mathrm{C}_{57} \mathrm{H}_{94} \mathrm{O}_{27}$ as determined by the observed (+)-HRESIMS protonated ion peak at $\mathrm{m} / \mathrm{z}$ $1233.5879[\mathrm{M}+\mathrm{Na}]^{+}$(calcd for $\mathrm{C}_{57} \mathrm{H}_{94} \mathrm{O}_{27} \mathrm{Na}, 1233.5875$ ). It showed a positive reaction to the Ehrlich's reagent (red color), suggesting a furostanol skeleton [20]. The ${ }^{13} \mathrm{C} \mathrm{NMR}$ spectrum (Table 1) displayed 57 carbons, of which 27 were assigned to the aglycone part and the remaining 30 were attributed to five hexose units. With the aid of the HSQC experiment, the ${ }^{1} \mathrm{H}$ and ${ }^{13} \mathrm{C}$ NMR spectrum (Table 1 ) attributable to the aglycone moiety showed resonances for four characteristic steroidal methyls at $\delta_{\mathrm{H}} 0.83(3 \mathrm{H}$, $\left.\mathrm{s}, \mathrm{CH}_{3}-19\right), 0.93\left(3 \mathrm{H}, \mathrm{d}, J=6.6 \mathrm{~Hz}, \mathrm{CH}_{3}-27\right), 1.00(3 \mathrm{H}, \mathrm{s}$, $\left.\mathrm{CH}_{3}-18\right)$, and $1.26\left(3 \mathrm{H}, \mathrm{d}, J=6.7 \mathrm{~Hz}, \mathrm{CH}_{3}-21\right)$, together with their corresponding carbons at $\delta_{\mathrm{C}} 16.3\left(\mathrm{CH}_{3}-19\right), 17.3$ $\left(\mathrm{CH}_{3}-27\right), 19.2\left(\mathrm{CH}_{3}-18\right), 16.3\left(\mathrm{CH}_{3}-21\right)$; two oxygenated methines at $\delta_{\mathrm{H}} 3.82(1 \mathrm{H}, \mathrm{m})$ and $4.88(1 \mathrm{H}, \mathrm{m})$, along with their corresponding carbons at $\delta_{\mathrm{C}} 77.8(\mathrm{CH}-3)$ and 80.8 $(\mathrm{CH}-16)$; an olefinic group at $\delta_{\mathrm{H}} 5.26(1 \mathrm{H}$, brs $)$ as well as $\delta_{\mathrm{C}} 121.6(\mathrm{CH}-6)$ and $140.6(\mathrm{C}-5)$; and a ketal carbon at $\delta_{\mathrm{C}} 110.4$ (C-22). The abovementioned data indicated that the aglycone of $\mathbf{1}$ should be a furostanol one as that of protodioscin (2) [21]. Moreover, the aglycone of $\mathbf{1}$ was further confirmed a by the following diagnostic ${ }^{1} \mathrm{H}-{ }^{1} \mathrm{H}$ COSY, HMBC, and ROESY correlations (Figs. 2 and 3). The ${ }^{1} \mathrm{H}-{ }^{1} \mathrm{H}$ COSY experiment revealed three structural fragments including $\mathrm{CH}_{2}-1-\mathrm{CH}_{2}-2-\mathrm{CH}-3-\mathrm{CH}_{2}-4, \mathrm{CH}-6-$ $\mathrm{CH}_{2}-7-\mathrm{CH}-8 /\left(-\mathrm{CH}-9-\mathrm{CH}_{2}-11-\mathrm{CH}_{2}-12\right) /-\mathrm{CH}-14-\mathrm{CH}_{2}-$ 15-CH-16-CH-17-CH-20- $\mathrm{CH}_{3}-21$, and $\mathrm{CH}_{2}-23-\mathrm{CH}_{2}-$ $24-\mathrm{CH}-25 /\left(-\mathrm{CH}_{3}-27\right) /-\mathrm{CH}_{2}-26$. Moreover, the observed $\mathrm{HMBC}$ from $\delta_{\mathrm{H}} 1.00\left(\mathrm{CH}_{3}-18\right)$ to $\delta_{\mathrm{C}} 39.7\left(\mathrm{CH}_{2}-12\right), 40.4$ (C-13), $56.4(\mathrm{CH}-14)$, and $63.6(\mathrm{CH}-17)$, from $\delta_{\mathrm{H}} 0.83$ $\left(\mathrm{CH}_{3}-19\right)$ to $\delta_{\mathrm{C}} 37.3\left(\mathrm{CH}_{2}-1\right), 140.6(\mathrm{C}-5), 50.1(\mathrm{CH}-$ $9)$, and $36.9(\mathrm{C}-10)$, and from both $\delta_{\mathrm{H}} 1.26\left(\mathrm{CH}_{3}-21\right)$ and $\delta_{\mathrm{H}} 2.00(\mathrm{H}-23 \mathrm{a})$ to $\delta_{\mathrm{C}} 110.4(\mathrm{C}-22)$ established the aglycone of 1 to be $22 \alpha$-hydroxyl-(25R)-furost- $\Delta^{5(6)}$ $3 \beta, 26$-diol. The ROESY correlations of $\delta_{\mathrm{H}} 1.00(\mathrm{Me}-$ $18)$ with $1.51(\mathrm{H}-8) / 2.17(\mathrm{H}-20) / 1.94(\mathrm{H}-23 \mathrm{~b})$ and of $\delta_{\mathrm{H}}$ 0.83 (Me-19) with $1.51(\mathrm{H}-8)$ and 1.68 (H-1a) verified these protons were placed at the same side, whereas the observed ROESY correlations of $\delta_{\mathrm{H}} 0.94(\mathrm{H}-1 \mathrm{~b})$ with 3.82 $(\mathrm{H}-3) / 0.86(\mathrm{H}-9)$, of $\delta_{\mathrm{H}} 1.02(\mathrm{H}-14)$ with $0.86(\mathrm{H}-9) / 1.87$ $(\mathrm{H}-17)$, and of $\delta_{\mathrm{H}} 1.87(\mathrm{H}-17)$ with $4.88(\mathrm{H}-16)$ indicated these protons were located at the other side. Additionally, the $25 R$ configuration of $\mathbf{1}$ was assigned according to the small chemical shift difference between Ha-26 and $\mathrm{Hb}-26$ at $\Delta \mathrm{ab}=0.34 \mathrm{ppm}(\Delta \mathrm{ab}>0.57 \mathrm{ppm}$ for $25 S$, and $\Delta \mathrm{ab}<0.48 \mathrm{ppm}$ for $25 R$ ) [22]. In view of aforementioned

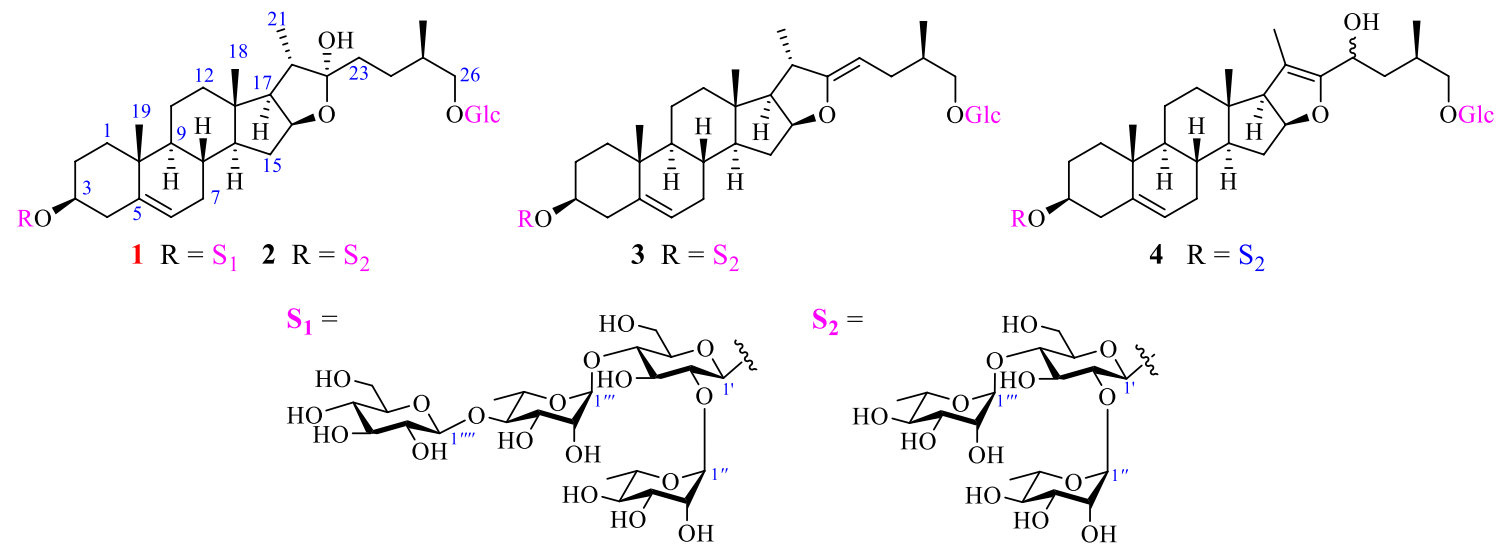

Fig. 1 Structures of 1-4 
Table $1{ }^{1} \mathrm{H}$ and ${ }^{13} \mathrm{C}$ NMR spectral data of 1 (600 and $150 \mathrm{MHz}$, pyridine- $\left.d_{5}\right)$

\begin{tabular}{|c|c|c|c|c|c|}
\hline \multirow[t]{2}{*}{ No } & \multicolumn{2}{|c|}{ Aglycone moiety } & \multirow[t]{2}{*}{ No } & \multicolumn{2}{|c|}{ Sugar moiety } \\
\hline & $\delta_{\mathrm{C}}$ & $\delta_{\mathrm{H}}($ mult., $J$ ) & & $\delta_{\mathrm{C}}$ & $\delta_{\mathrm{H}}($ mult., $J)$ \\
\hline \multirow[t]{2}{*}{1} & $37.3, \mathrm{CH}_{2}$ & $\begin{array}{l}\text { a } 1.68 \mathrm{~m} \\
\text { b } 0.94 \mathrm{~m}\end{array}$ & 3-O-Glc & & \\
\hline & & & $1^{\prime}$ & $100.1, \mathrm{CH}$ & $4.88 \mathrm{~d}(7.7)$ \\
\hline 2 & $29.9, \mathrm{CH}_{2}$ & $\begin{array}{l}\text { a } 1.98 \mathrm{~m} \\
\text { b } 1.79 \mathrm{~m}\end{array}$ & $2^{\prime}$ & $77.6, \mathrm{CH}$ & $4.10 \mathrm{~m}$ \\
\hline 3 & $77.8, \mathrm{CH}$ & $3.82 \mathrm{~m}$ & $3^{\prime}$ & $73.8, \mathrm{CH}$ & $4.26 \mathrm{~m}$ \\
\hline 4 & $38.7, \mathrm{CH}_{2}$ & $\begin{array}{l}\text { a } 2.71 \mathrm{~m} \\
\text { b } 2.64 \mathrm{~m}\end{array}$ & $4^{\prime}$ & 77.1, CH & $4.31 \mathrm{~m}$ \\
\hline 5 & $140.6, \mathrm{C}$ & & $5^{\prime}$ & $76.7, \mathrm{CH}$ & $4.30 \mathrm{~m}$ \\
\hline 6 & $121.6, \mathrm{CH}$ & 5.26 br s & $6^{\prime}$ & $61.0, \mathrm{CH}_{2}$ & $\begin{array}{l}\text { a } 4.12 \mathrm{~m} \\
\text { b } 3.98 \mathrm{~m}\end{array}$ \\
\hline 7 & $32.1, \mathrm{CH}_{2}$ & $1.832 \mathrm{H} \mathrm{m}$ & & & \\
\hline 8 & $31.5, \mathrm{CH}$ & $1.51 \mathrm{~m}$ & $2^{\prime}-O$-Rha & & \\
\hline 9 & $50.1, \mathrm{CH}$ & $0.86 \mathrm{~m}$ & $1^{\prime \prime}$ & 101.6, CH & 6.27 br s \\
\hline 10 & $36.9, \mathrm{C}$ & & $2^{\prime \prime}$ & $71.4, \mathrm{CH}$ & $4.76 \mathrm{~m}$ \\
\hline 11 & $20.9, \mathrm{CH}_{2}$ & $1.382 \mathrm{H} \mathrm{m}$ & $3^{\prime \prime}$ & $72.5, \mathrm{CH}$ & $4.75 \mathrm{~m}$ \\
\hline 12 & $39.7, \mathrm{CH}_{2}$ & $\begin{array}{l}\text { a } 1.70 \mathrm{~m} \\
\text { b } 1.06 \mathrm{~m} \\
\text { b1.06 m }\end{array}$ & $4^{\prime \prime}$ & $73.8, \mathrm{CH}$ & $4.26 \mathrm{~m}$ \\
\hline 13 & $40.4, \mathrm{C}$ & & $5^{\prime \prime}$ & $69.3, \mathrm{CH}$ & $4.84 \mathrm{~m}$ \\
\hline 14 & $56.4, \mathrm{CH}$ & $1.02 \mathrm{~m}$ & $6^{\prime \prime}$ & $18.4, \mathrm{CH}_{3}$ & $1.683 \mathrm{H} \mathrm{d}(6.0)$ \\
\hline 15 & $32.2, \mathrm{CH}_{2}$ & $1.402 \mathrm{H} \mathrm{m}$ & $4^{\prime}-O-\mathrm{Rha}$ & & \\
\hline 16 & $80.8, \mathrm{CH}$ & $4.88 \mathrm{~m}$ & $1^{\prime \prime \prime}$ & $101.8, \mathrm{CH}$ & $5.74 \mathrm{br} \mathrm{s}$ \\
\hline 17 & $63.6, \mathrm{CH}$ & $1.87 \mathrm{~m}$ & $2^{\prime \prime \prime}$ & 71.7, CH & $4.76 \mathrm{~m}$ \\
\hline 18 & $19.2, \mathrm{CH}_{3}$ & $1.003 \mathrm{H} \mathrm{s}$ & $3^{\prime \prime \prime}$ & $72.2, \mathrm{CH}$ & $4.59 \mathrm{~m}$ \\
\hline 19 & $16.3, \mathrm{CH}_{3}$ & $0.833 \mathrm{H} \mathrm{s}$ & $4^{\prime \prime \prime}$ & $84.9, \mathrm{CH}$ & $4.35 \mathrm{~m}$ \\
\hline 20 & $40.6, \mathrm{CH}$ & $2.17 \mathrm{~m}$ & $5^{\prime \prime \prime}$ & $68.3, \mathrm{CH}$ & $4.93 \mathrm{~m}$ \\
\hline 21 & $16.3, \mathrm{CH}_{3}$ & $1.263 \mathrm{H} \mathrm{d}(6.7)$ & $6^{\prime \prime \prime}$ & $18.2, \mathrm{CH}_{3}$ & $1.603 \mathrm{H} \mathrm{d}(6.0)$ \\
\hline 22 & $110.4, \mathrm{C}$ & & 4"-O-Glc & & \\
\hline 23 & $36.9, \mathrm{CH}_{2}$ & $\begin{array}{l}\text { a } 2.00 \mathrm{~m} \\
\text { b } 1.94 \mathrm{~m} \\
\text { b } 1.94 \mathrm{~m}\end{array}$ & $1^{\prime \prime \prime}$ & $106.4, \mathrm{CH}$ & $5.14 \mathrm{~d}(7.7)$ \\
\hline 24 & 28.1, $\mathrm{CH}_{2}$ & $\begin{array}{l}\text { a } 1.97 \mathrm{~m} \\
\text { b } 1.63 \mathrm{~m}\end{array}$ & $2^{\prime \prime \prime \prime}$ & 76.7, CH & $3.98 \mathrm{~m}$ \\
\hline 25 & $34.0, \mathrm{CH}$ & $1.93 \mathrm{~m}$ & $3 " \prime \prime$ & $78.2, \mathrm{CH}$ & $3.70 \mathrm{~m}$ \\
\hline 26 & 74.9, $\mathrm{CH}_{2}$ & $\begin{array}{l}\text { a } 3.55 \mathrm{dd}(9.0,6.1) \\
\text { b } 3.88 \mathrm{~m}\end{array}$ & $4^{\prime \prime \prime}$ & 71.0, $\mathrm{CH}$ & $4.12 \mathrm{~m}$ \\
\hline \multirow[t]{9}{*}{27} & $17.3, \mathrm{CH}_{3}$ & $0.933 \mathrm{H} \mathrm{d}(6.6)$ & $5^{\prime \prime \prime}$ & $76.3, \mathrm{CH}$ & $4.00 \mathrm{~m}$ \\
\hline & & & $6^{\prime \prime \prime}$ & $62.2, \mathrm{CH}_{2}$ & $\begin{array}{l}\text { a } 4.45 \mathrm{~d}(12.4) \\
\text { b } 4.28 \mathrm{~m}\end{array}$ \\
\hline & & & 26- $O-\mathrm{Glc}$ & & \\
\hline & & & $1^{\prime \prime \prime \prime \prime}$ & 104.6, CH & $4.73 \mathrm{~d}(7.8)$ \\
\hline & & & $2^{\prime \prime \prime \prime \prime}$ & $75.0, \mathrm{CH}$ & $3.82 \mathrm{~m}$ \\
\hline & & & $3^{\prime \prime \prime \prime \prime}$ & $78.2, \mathrm{CH}$ & $3.99 \mathrm{~m}$ \\
\hline & & & $4^{\prime \prime \prime \prime \prime}$ & $71.7, \mathrm{CH}$ & $4.12 \mathrm{~m}$ \\
\hline & & & $5^{\prime \prime \prime \prime \prime}$ & $78.3, \mathrm{CH}$ & $4.10 \mathrm{~m}$ \\
\hline & & & $6^{\prime \prime \prime \prime \prime}$ & $62.5, \mathrm{CH}_{2}$ & $\begin{array}{l}\text { a } 4.45 \mathrm{~m} \\
\text { b } 4.28 \mathrm{~m}\end{array}$ \\
\hline
\end{tabular}




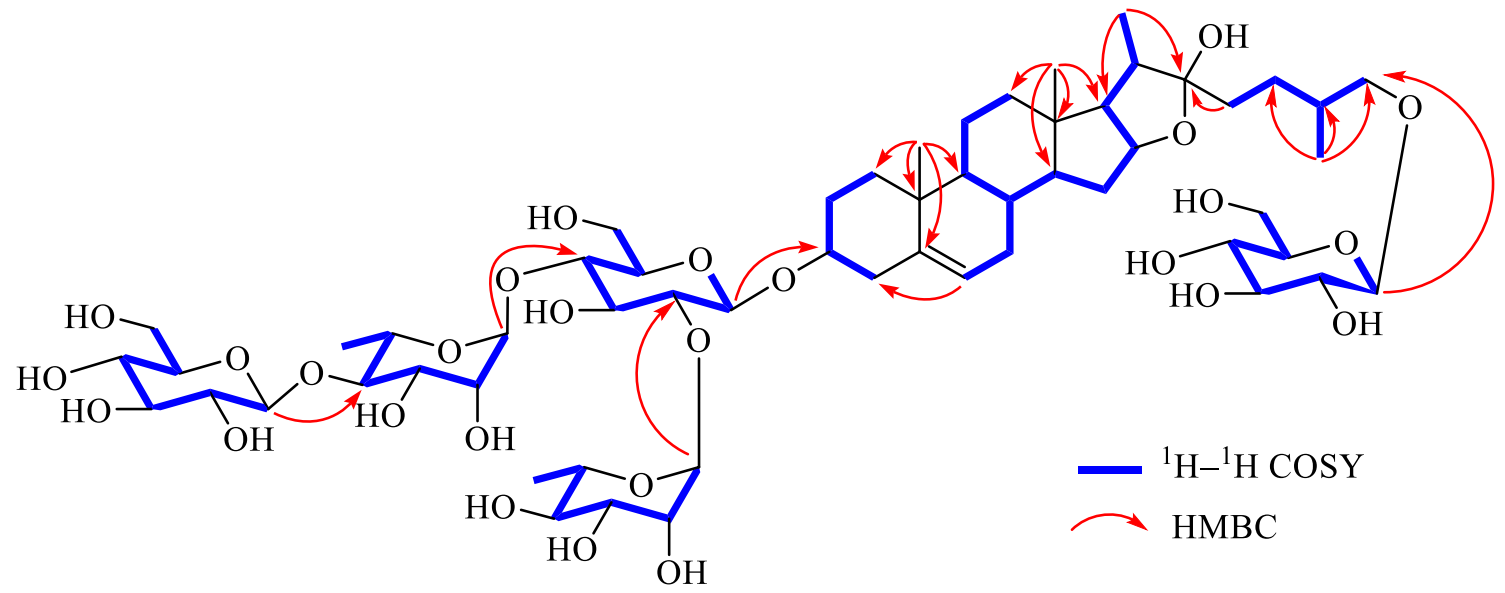

Fig. 2 Key ${ }^{1} \mathrm{H}-{ }^{1} \mathrm{H}$ COSY and HMBC correlations of $\mathbf{1}$

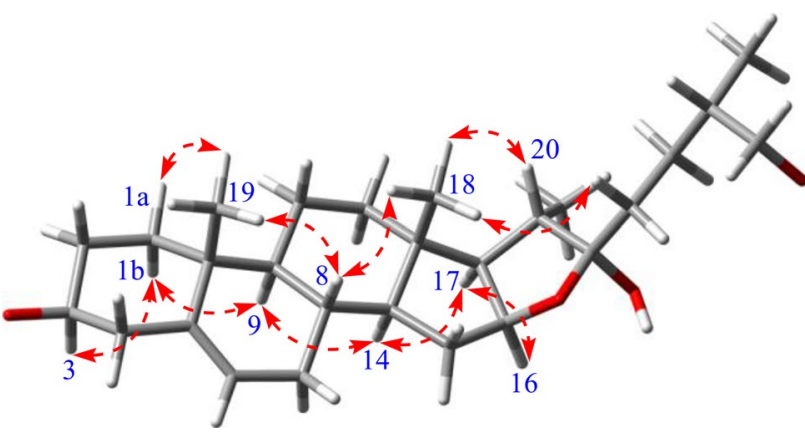

Fig. 3 Key ROESY correlations for the aglycone moiety of $\mathbf{1}$

evidence, the aglycone of $\mathbf{1}$ was thus elucidated as $22 \alpha$-hydroxyl-(25R)-furost- $\Delta^{5(6)}-3 \beta, 26$-diol.

As for the sugar units of $\mathbf{1}$, its ${ }^{1} \mathrm{H}$ NMR spectrum (Table 1) displayed the presence of five anomeric proton signals at $\delta_{\mathrm{H}}$ $4.73\left(1 \mathrm{H}, \mathrm{d}, J=7.8 \mathrm{~Hz}, \mathrm{H}-1^{\prime \prime \prime} "\right), 4.88(1 \mathrm{H}, \mathrm{d}, J=7.7 \mathrm{~Hz}$, $\left.\mathrm{H}-1^{\prime}\right), 5.14\left(1 \mathrm{H}, \mathrm{d}, J=7.7 \mathrm{~Hz}, \mathrm{H}-1^{\prime \prime \prime}\right), 5.74\left(1 \mathrm{H}, \mathrm{brs}, \mathrm{H}-1^{\prime \prime \prime}\right)$ and $6.27\left(1 \mathrm{H}\right.$, brs, $\left.\mathrm{H}-1{ }^{\prime \prime}\right)$, which showed correlations in the HSQC spectrum with five anomeric carbons at $\delta_{\mathrm{C}} 104.6$ (CH-1"'" $), 100.1\left(\mathrm{CH}-1^{\prime}\right), 106.4\left(\mathrm{CH}-1^{\prime \prime \prime}\right), 101.8\left(\mathrm{CH}-1^{\prime \prime \prime}\right)$, and $101.6\left(\mathrm{CH}-1^{\prime \prime}\right)$. With the assistance of MS spectrum, the sugar moiety of $\mathbf{1}$ was preliminary determined. Specifically, the $[\mathrm{M}-\mathrm{H}]^{-}$ion $(\mathrm{m} / \mathrm{z}, 1209.6)$ displayed $\mathbf{1}$ had a molecular weight (MW) of 1210.6 Da in the negative ion mode of ESI$\mathrm{MS}^{\mathrm{n}}$. The observed ions with $\mathrm{m} / \mathrm{z}$ values of $1047.5,901.5$, and 755.4 indicated the sequential cleavage of two rhamnopyranosyl units followed by the cleavage of a glucopyranosyl moiety from the parent $[\mathrm{M}-\mathrm{H}]^{-}$ion $(\mathrm{m} / \mathrm{z}, 1209.6)$, respectively. Likewise, the $\mathrm{MS}^{2}$ spectrum also afforded $\mathrm{m} / \mathrm{z}$ value of 593.4 that was indicative of the loss of one glucopyranosyl group from the C-3 position or the C-26 position (Scheme 1). Also, acid hydrolysis of $\mathbf{1}$ also gave D-glucoses and L-rhamnoses as the sugar residue, which was confirmed by HPLC analysis of their corresponding PMP derived adducts. All the anomeric protons of D-glucose possessed $\beta$-configurations due to their ${ }^{3} J_{\mathrm{H} 1, \mathrm{H} 2}$ coupling constants (7.8, 7.7, and $7.7 \mathrm{~Hz}$ ), and both anomeric protons of L-rhamnoses shared $\alpha$-configurations due to the chemical shifts of C-3 $\left(\delta_{\mathrm{C}} 72.5\right.$ and 72.2$)$ and $\mathrm{C}-5\left(\delta_{\mathrm{C}} 69.3\right.$ and 68.3$)$, respectively. In the HMBC spectrum, the long-range correlations from $\delta_{\mathrm{H}} 4.88\left(\mathrm{H}-1^{\prime}\right)$ to $\delta_{\mathrm{C}} 77.8(\mathrm{CH}-3)$, from $\delta_{\mathrm{H}} 4.73\left(\mathrm{H}-1^{\prime \prime \prime}{ }^{\prime \prime}\right)$ to $\delta_{\mathrm{C}} 74.9\left(\mathrm{CH}_{2}-26\right)$, from $\delta_{\mathrm{H}} 6.27\left(\mathrm{H}-1^{\prime \prime}\right)$ to $\delta_{\mathrm{C}} 77.6\left(\mathrm{CH}-2^{\prime}\right)$, from $\delta_{\mathrm{H}} 5.74\left(\mathrm{H}-1^{\prime \prime \prime}\right)$ to $\delta_{\mathrm{C}} 77.1\left(\mathrm{CH}-4^{\prime}\right)$, and from $\delta_{\mathrm{H}} 5.14$ $\left(\mathrm{H}-1^{\prime \prime \prime}\right)$ to $\delta_{\mathrm{C}} 84.9$ (CH-4"') established the sequence for 3 - $O$-sugar chain as an $[\alpha$-L-rhamnopyranosyl- $(1 \rightarrow 2)]$-[ $\beta$-Dglucopyranosyl-( $1 \rightarrow 4)$ - $\alpha$-L-rhamnopyranosyl- $(1 \rightarrow 4)]$ - $\beta$-Dglucopyranosyl moiety and for $26-O$-sugar chain as $\beta$-Dglucopyranosyl moiety, respectively. Based on the above information presented, the structure of $\mathbf{1}$ was thus elucidated to be $26-O-\beta$-D-glucopyranosyl-22 $\alpha$-hydroxyl- $(25 R)-\Delta^{5(6)}$ furost-3 $\beta, 26$-diol-3- $O$ - $\alpha$-L-rhamnopyranosyl- $(1 \rightarrow 2)$-[ $\beta$-Dglucopyranosyl-( $1 \rightarrow 4)$ - $\alpha$-L-rhamnopyranosyl-( $1 \rightarrow 4)]-\beta$-Dglucopyranoside.

Additionally, three known steroidal glycosides were identified as protodioscin (2) [21], (25R)-26-O$\beta$-D-glucopyranosyl-3 $\beta, 20 \alpha, 26$-trihydroxyfurostan-5, 22 -diene-3- $O$ - $\alpha$-L-rhamnopyranosyl- $(1 \rightarrow 2)$ - $[\alpha$-Lrhamnopyranosyl-(1 $\rightarrow 4)$ ]- $O$ - $\beta$-D-glucopyranoside (3) [23], and dioscoreside $\mathrm{H}(4)$ [24] by comparison of their spectroscopic data with those reported in the literatures.

The steroid saponins obtained from species of Liliaceae have shown the potential to significantly inhibit the proliferations of various human tumor cell lines in vitro [25-29]. Therefore, all isolated compounds were evaluated for their cytotoxicity against MHCC $97 \mathrm{H}$ and $\mathrm{H} 1299$ by the MTT method. More specifically, compared with the $\mathrm{IC}_{50}$ values of positive control doxorubicin hydrochloride, and both $\mathbf{1}$ and $\mathbf{2}$ displayed strong cytotoxicity against MHCC97H and H1299 cells with $\mathrm{IC}_{50}$ values of $3.56 \pm 0.45 / 4.18 \pm 0.43 \mu \mathrm{g} / \mathrm{mL}$ and 

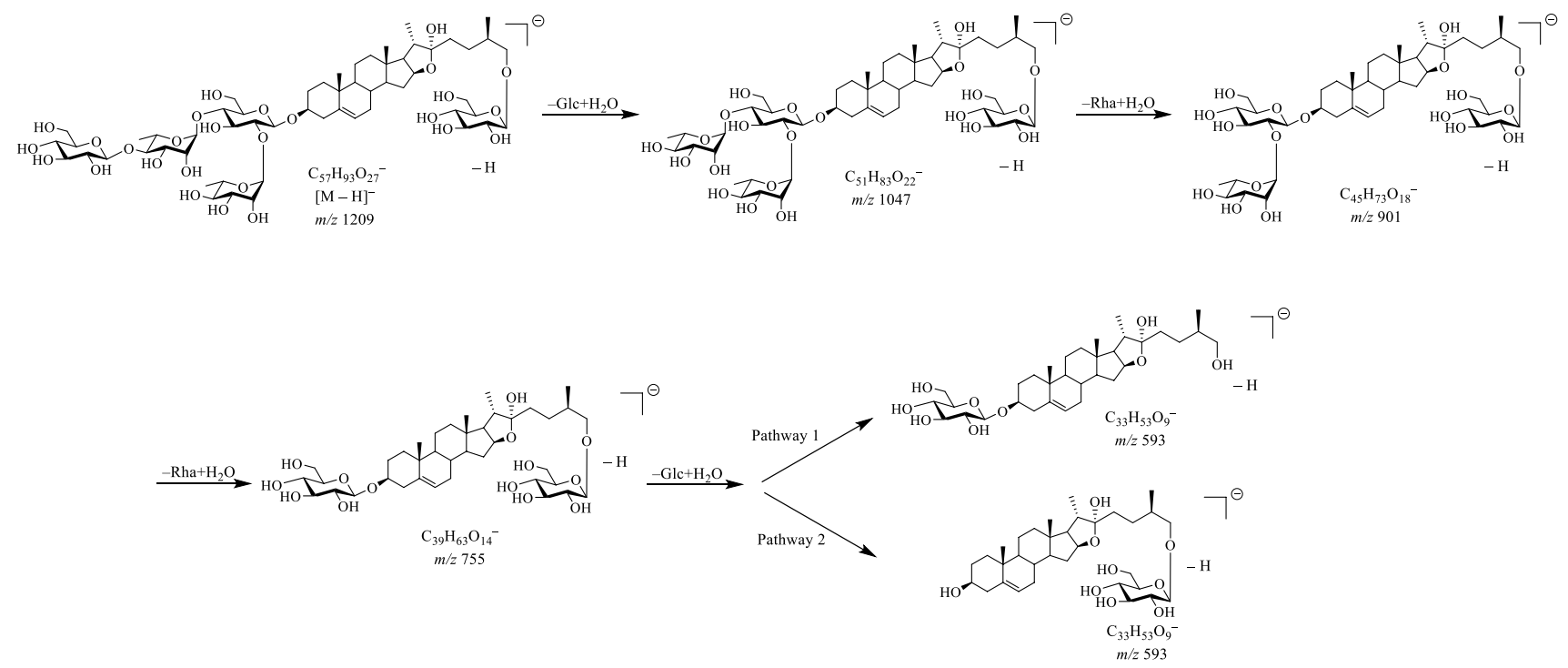

Scheme 1 The fragmentation process of 1 in the ESI- MS negative scan

$5.26 \pm 0.74 / 4.15 \pm 0.59 \mu \mathrm{g} / \mathrm{mL}$, respectively (see Fig. 4). Furthermore, as can be seen from Fig. 4, compared with the positive control doxorubicin hydrochloride, saponins $\mathbf{1}$ and $\mathbf{2}$ could significantly inhibit their proliferation (Table 2).

Moreover, all obtained steroid saponins were evaluated for their antimicrobial activity against Escherichia coli (ML35P), Bacillus cereus (CMCC(B) 63303), Candida albicans (ATCC 2091), Bacillus subtilis (ATCC 6633), Streptococcus hemolyticus (ATCC 19615), Listeria monocytogenes (ATCC 19114), Pseudomonas aeruginosa (PO01), Staphylococcus aureus (ATCC 4330), Salmonella Typhimurium (SL1344) and Staphylococcus epidermidis (CMCC 26069) by the microdilution broth susceptibility assay. The results (see Table 3) revealed that saponins $\mathbf{1} \mathbf{- 4}$ showed moderate antimicrobial activity against $C$. albicans and B. subtilis, while only saponin $\mathbf{3}$ showed weak antimicrobial activity against S. aureus $(63.30 \pm 0.55 \mu \mathrm{g} / \mathrm{mL})$.

\section{Experimental}

\subsection{General Experiment Procedures}

Optical rotation was measured on a Autopol VI automatic polarimeter. The IR spectrum were measured on a Thermo Nicolet iS10 infrared spectrophotometer with KBr disk. The NMR spectra were obtained on Bruker DRX-400 and DRX-600 spectrometers. Chemical shifts $(\delta)$ were expressed in ppm with reference to the solvent signals. Both ESI and HRESIMS spectra were performed on an UPLC-IT-TOF spectrometer. Semi-preparative HPLC was performed on a Waters 600 with a COSMOSIL C18 $(10 \times 250 \mathrm{~mm}$, Nacalai Tesque Corporation, Japan) column. Analytical HPLC was performed on a Shimadzu SIL-20A Series HPLC system equipped with a reverse-phase COSMOSIL C18 column (4.6 $\mathrm{mm} \times 250 \mathrm{~mm}, 5 \mu \mathrm{m}$, Nacalai Tesque Corporation, Japan). Column chromatography was carried out using silica gel (100-200 mesh, Qingdao Haiyang Chemical, Qingdao, Co., Ltd., People's Republic of China) and macro-porous absorption resin (D101, Donghong Chemical Co., Ltd., People's Republic of China). The PMP (Chengdu Aikeda Chemical Reagent Co., Ltd., China) was purchased from Beijing 4A Biotech Co., Ltd. (Beijing, China). Fractions were monitored by TLC, and spots were visualized by heating silica gel plates sprayed with Ehrlich's reagent.

\subsection{Plant Materials}

The roots of A. cochinchinensis was purchased from 'Luosiwan' Chinese herbal medicine Market, Kunming, Yunnan Province, in November 2019, identified by Dr. Xu-Jie Qin. A voucher specimen (No. Luo 20191106) has been deposited at State Key Laboratory of Phytochemistry and Plant Resource in West China, Kunming Institute of Botany, Chinese Academy of Sciences.

\subsection{Extraction and Isolation}

The air-dried roots of $A$. cochinchinensis $(5.0 \mathrm{~kg})$ were extracted with $90 \%$ aqueous $\mathrm{EtOH}$ at $80{ }^{\circ} \mathrm{C}(15 \mathrm{~L} \times 4$, each time for $3 \mathrm{~h}$ ). The solvent was removed under reduced pressure to yield an amber residue $(2.5 \mathrm{~kg})$. The residue was subjected to column chromatography over an macroporous resin column eluted first with $\mathrm{H}_{2} \mathrm{O}$ then successively with $25 \%$, $70 \%$, and $90 \% \mathrm{EtOH}$, respectively. The $70 \% \mathrm{EtOH}$ partition was evaporated under reduced pressure to obtain a total 
$1 \mathrm{MHCC} 97 \mathrm{H} 72 \mathrm{~h}$

(A)

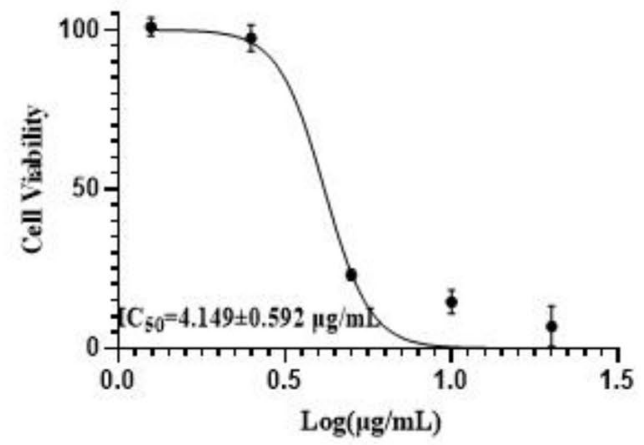

$1 \mathrm{H1299} 72 \mathrm{~h}$

(B)

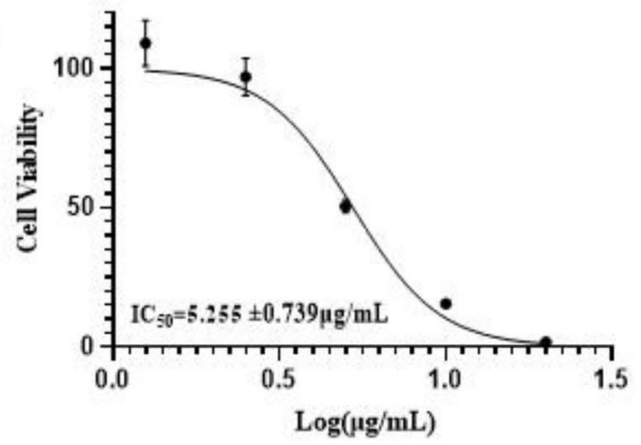

(C)

$\mu \mathrm{g} / \mathrm{mL}$

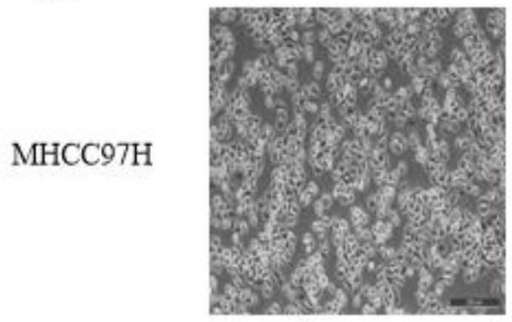

Control

0

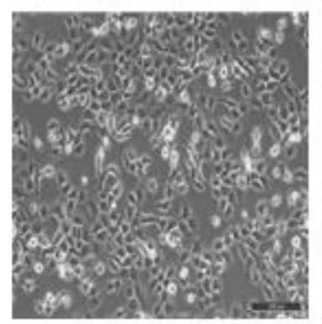

Positive control

10
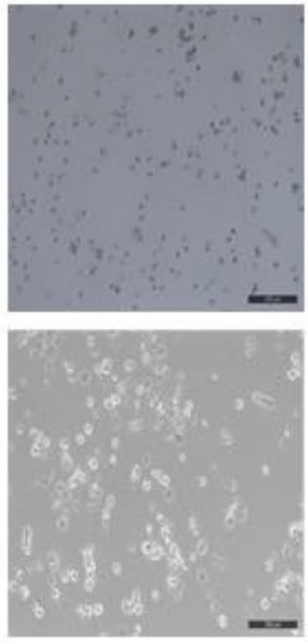

$2 \mathrm{MHCC} 97 \mathrm{H} 72 \mathrm{~h}$

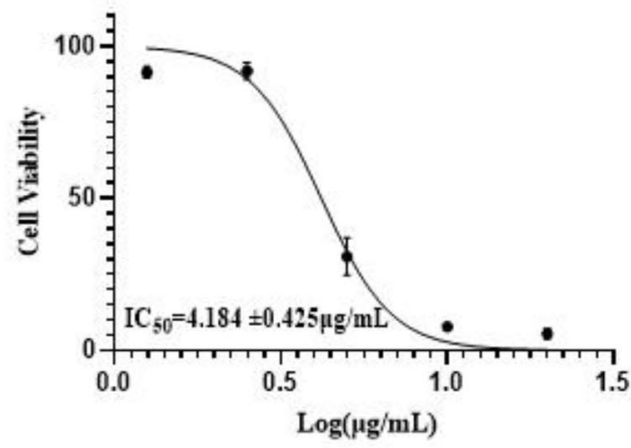

$2 \mathrm{H1299} 72 \mathrm{~h}$

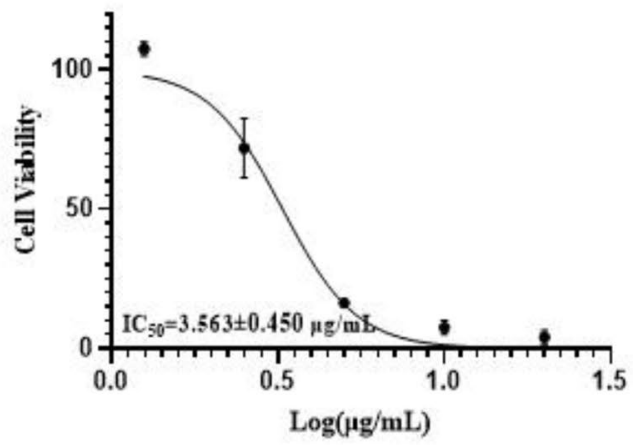

2

10

10
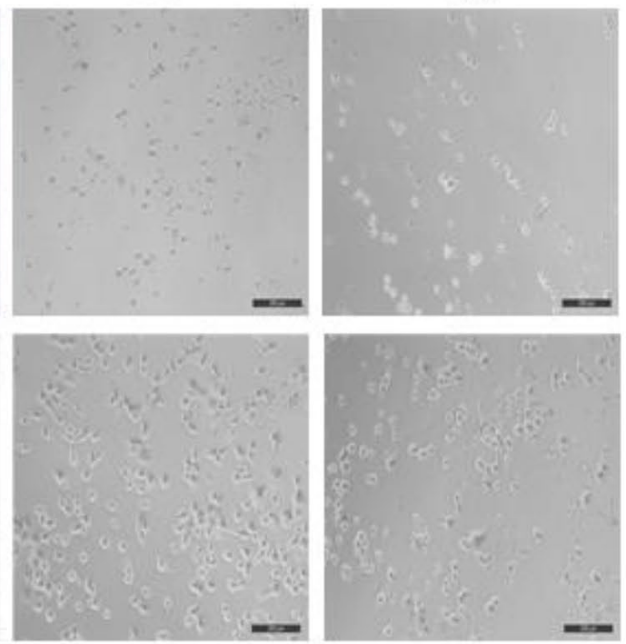

Fig. 4 Effects of $\mathbf{1}$ and $\mathbf{2}$ on MHCC97H and H1299 cells proliferation $(n=3)$. A The $\mathrm{IC}_{50}$ values of $\mathbf{1}$ and $\mathbf{2}$ against MHCC97H; B The IC 50 values of $\mathbf{1}$ and $\mathbf{2}$ against H1299; C Inhibition effects of MHCC97H and H1299 cells proliferation by $\mathbf{1}$ and $\mathbf{2}$ after cultivation for $72 \mathrm{~h}$

steroidal saponin moiety. The total saponins ( $153 \mathrm{~g}$ ) was subjected to a silica gel column eluting with a $\mathrm{CHCl}_{3}-\mathrm{MeOH}-$ $\mathrm{H}_{2} \mathrm{O}$ gradient $(80: 20: 2 \rightarrow 65: 35: 10)$ to yield five fractions (Fr. A-Fr. E). Fraction C (105 g) was chromatographed on a silica gel column $\left(\mathrm{CHCl}_{3}-\mathrm{MeOH}-\mathrm{H}_{2} \mathrm{O}, 9: 1: 0.1\right)$ to give saponin 2 (70 g) and Fr. C1. Fr. C1 (230.5 mg) was further purified by semi-preparative HPLC to afford $1(29.8 \mathrm{mg}$; $\left.t_{\mathrm{R}}=12 \mathrm{~min} ; \mathrm{MeCN}-\mathrm{H}_{2} \mathrm{O}, 28: 72,3.0 \mathrm{~mL} / \mathrm{min}\right)$. Fraction D (12 g) was separated on a silica gel column $\left(\mathrm{CHCl}_{3}-\right.$ $\left.\mathrm{MeOH}-\mathrm{H}_{2} \mathrm{O}, 8: 2: 0.2\right)$ and then purified by semi-preparative 
Table 2 Cytotoxicity of saponins 1 and $2\left(\mathrm{IC}_{50} \pm \mathrm{SD}, \mu \mathrm{g} / \mathrm{mL}\right)$

\begin{tabular}{llc}
\hline Compound & H1299 & MHCC97H \\
\hline $\mathbf{1}$ & $5.26 \pm 0.74$ & $3.56 \pm 0.45$ \\
$\mathbf{2}$ & $4.15 \pm 0.59$ & $4.18 \pm 0.43$ \\
Doxorubicin hydrochloride $^{\mathrm{a}}$ & $0.86 \pm 0.39$ & $0.20 \pm 0.08$ \\
\hline
\end{tabular}

${ }^{\text {a Positive control }}$

Table 3 Antimicrobial activity of saponins $1-4\left(\mathrm{IC}_{50} \pm \mathrm{SD}, \mu \mathrm{g} / \mathrm{mL}\right)$

\begin{tabular}{llll}
\hline Compound & C. albicans & B. subtilis & S. aureus \\
\hline $\mathbf{1}$ & $55.11 \pm 0.32$ & $47.93 \pm 0.18$ & $\mathrm{NA}^{\mathrm{a}}$ \\
$\mathbf{2}$ & $72.05 \pm 0.49$ & $69.30 \pm 0.16$ & $\mathrm{NA}^{\mathrm{a}}$ \\
$\mathbf{3}$ & $52.05 \pm 0.31$ & $47.19 \pm 0.19$ & $63.30 \pm 0.55$ \\
$\mathbf{4}$ & $52.05 \pm 0.31$ & $30.07 \pm 0.22$ & $\mathrm{NA}^{\mathrm{a}}$ \\
Streptomycin sulfat $^{\mathrm{a}}$ & $40.88 \pm 0.33$ & $93.49 \pm 0.50$ & $22.97 \pm 0.24$ \\
\hline
\end{tabular}

$N A$ no activity $(>100 \mu \mathrm{g} / \mathrm{mL})$

${ }^{\text {a }}$ Positive control

HPLC to yield saponins 3 (3.4 mg, $t_{\mathrm{R}}=20.5 \mathrm{~min}$; MeCN$\left.\mathrm{H}_{2} \mathrm{O}, 35: 65,1.0 \mathrm{~mL} / \mathrm{min}\right)$ and $4\left(2.6 \mathrm{mg}, t_{\mathrm{R}}=23.5 \mathrm{~min}\right.$; $\left.\mathrm{CH}_{3} \mathrm{CN}-\mathrm{H}_{2} \mathrm{O}, 35: 65,1.0 \mathrm{~mL} / \mathrm{min}\right)$.

\subsection{Spectroscopic Data of 1}

$26-O-\beta$-D-glucopyranosyl-22 $\alpha$-hydroxyl- $(25 R)-\Delta^{5(6)}$ furost-3 $\beta, 26$-diol-3- $O$ - $\alpha$-L-rhamnopyranosyl- $(1 \rightarrow 2)$-[ $\beta$-Dglucopyranosyl-( $1 \rightarrow 4)-\alpha$-L-rhamnopyranosyl- $(1 \rightarrow 4)]-\beta$-Dglucopyranoside (1): white amorphous powder, $[\alpha]-46.86$ $(c 0.11, \mathrm{MeOH})$; IR $\left(\nu_{\max }\right): 3417,2933,2851,1635,1453$, $1382,1045 \mathrm{~cm}^{-1}$; HRESIMS $\mathrm{m} / \mathrm{z} 1233.5879[\mathrm{M}+\mathrm{Na}]^{+}$ (calcd for $\mathrm{C}_{57} \mathrm{H}_{94} \mathrm{O}_{27} \mathrm{Na}, 1233.5875$ ). ${ }^{1} \mathrm{H}$ (pyridine- $d_{5}$, $600 \mathrm{MHz}$ ) and ${ }^{13} \mathrm{C}$ (pyridine- $d_{5}, 150 \mathrm{MHz}$ ) NMR spectral data, see Table 1.

\subsection{Acid Hydrolysis of 1}

The acid hydrolysis of compound $\mathbf{1}$ was carried out by a previously reported procedure [19]. Compound $\mathbf{1}(2.0 \mathrm{mg})$ was refluxed at $120^{\circ} \mathrm{C}$ for $2 \mathrm{~h}$ with $2 \mathrm{M}$ TFA on an oil bath. The aglycone was removed by the extraction with $\mathrm{CHCl}_{3}$ $(5.0 \mathrm{~mL})$ for three times. The reaction residue was filtered after neutralizing with $60.0 \mu \mathrm{L}$ of $\mathrm{NaOH}(0.3 \mathrm{M})$. After removing the solvent under reduced pressure, the residue was refluxed at $75^{\circ} \mathrm{C}$ for $1 \mathrm{~h}$ with $60.0 \mu \mathrm{L}$ of PMP $(0.5 \mathrm{M}$ in methanol). Moreover, the reaction was quenched with 60.0 $\mu \mathrm{L}$ of $\mathrm{HCl}(0.3 \mathrm{M})$ and the reaction mixture was extracted with $\mathrm{CHCl}_{3}(5.0 \mathrm{~mL}$, three times). Then, the aqueous layer was analyzed over HPLC (18\% acetonitrile: $82 \%$ sodium phosphate ( $\mathrm{pH} 6.8 ; 1.5 \mathrm{~mL} / \mathrm{min})$. Likewise, the standard monosaccharides D-glucose $(1.0 \mathrm{mg})$ and L-rhamnose
$(1.0 \mathrm{mg})$ were derivatized with PMP by the same way as $\mathbf{1}$, and HPLC analyses were performed under the same conditions as $\mathbf{1}$. The sugar units in $\mathbf{1}$ were identified as D-glucose $\left(t_{\mathrm{R}}=14.5 \mathrm{~min}\right)$ and L-rhamnose $\left(t_{\mathrm{R}}=17.0 \mathrm{~min}\right)$ by comparison of the retention times of the corresponding derivatives.

\subsection{Cytotoxicity Assay}

The cytotoxicity of isolated compounds was determined to use the MTT method with a slight modification [30]. Briefly, two human cancer (MHCC97H and H1299) cell lines were incubated in 96-well plates at a density of $2 \times 10^{3}$ cells/well in DMEM medium supplemented with $10 \%$ fetal bovine serum at $37{ }^{\circ} \mathrm{C}$ with $5 \% \mathrm{CO}_{2}$. After overnight incubation, cells were treated with tested compounds at different concentrations $(20.00,10.00,5.00,2.50$, and $1.25 \mu \mathrm{g} / \mathrm{mL})$ for $72 \mathrm{~h}$. Subsequently, the culture mediums were exchanged by DMEM medium which contained $10 \%$ MTS reagent [3-(4,5-dimethylthiazol-2-yl)-5-(3-carboxymethoxyphenyl)2-(4-sulfophenyl)-2H-tetrazolium, inner salt] and then cultured for another $4 \mathrm{~h}$. The absorbance was recorded on a microplate reader at $490 \mathrm{~nm}$.

\subsection{Antimicrobial Activity Assay}

The antimicrobial activity of isolated steroid saponins against 10 strains using the microdilution broth susceptibility assay [31]. The strains frozen in the refrigerator at -80 ${ }^{\circ} \mathrm{C}$ were activated and inoculated on standard tryptone soy broth agar (TSA) plates at $37{ }^{\circ} \mathrm{C}$ for $8 \mathrm{~h}$ to observe the bacterial growth. Subsequently, single colonies were selected and inoculated in tryptone soy broth (TSB) plates. After cultivated at $37{ }^{\circ} \mathrm{C}$ in shaker $(120 \mathrm{rpm})$ for $8 \mathrm{~h}$, the absorbance of bacterial solution was measured and its concentration was adjusted to $10^{5} \mathrm{CFU} / \mathrm{mL}$. Whereafter, an inoculum of $10^{5} \mathrm{CFU} / \mathrm{mL}$ was made to sterile 96 -well plate containing tested compounds at different concentrations $(100.00,50.00$, $25.00,12.50,6.25$ and $3.13 \mu \mathrm{g} / \mathrm{mL}$ ) at $37{ }^{\circ} \mathrm{C}$ for $8 \mathrm{~h}$. The wells containing only broth served as growth control. The absorbance of bacterial solution was recorded on a microplate reader at $600 \mathrm{~nm}$.

\section{Conclusion}

In summary, a chemical examination of the roots of $A$. cochinchinensis led to the identification of one new furostanol glycoside $26-O-\beta$-D-glucopyranosyl$22 \alpha$-hydroxyl-(25R)- $\Delta^{5(6)}$-furost-3 $\beta, 26$-diol-3- $O-\alpha$-Lrhamnopyranosyl-( $1 \rightarrow 2)$-[ $\beta$-D-glucopyranosyl-( $1 \rightarrow 4)-\alpha$-Lrhamnopyranosyl- $(1 \rightarrow 4)]-\beta$-D-glucopyranoside $(\mathbf{1})$ and three known one (2-4). Meanwhile, compounds 1 and 2 exhibited cytotoxic and anti-proliferative effects on two 
human (MHCC97H and H1299) cancer cell lines. At the same time, compounds 1-4 displayed moderate antimicrobial activity against $C$. albicans and B. subtilis, and compound $\mathbf{3}$ displayed weak antimicrobial activity against $S$. aureus.

Supplementary Information The online version contains supplementary material available at https://doi.org/10.1007/s13659-021-00321-0.

Acknowledgements This study was financially supported by the National Natural Science Foundation of China (Grant Nos. 31770388 and U1802281) and the Second Tibetan Plateau Scientific Expedition and Research (STEP) program (Grant No. 2019QZKK0502).

\section{Declarations}

Conflict of interest The authors declare no competing financial interest.

Open Access This article is licensed under a Creative Commons Attribution 4.0 International License, which permits use, sharing, adaptation, distribution and reproduction in any medium or format, as long as you give appropriate credit to the original author(s) and the source, provide a link to the Creative Commons licence, and indicate if changes were made. The images or other third party material in this article are included in the article's Creative Commons licence, unless indicated otherwise in a credit line to the material. If material is not included in the article's Creative Commons licence and your intended use is not permitted by statutory regulation or exceeds the permitted use, you will need to obtain permission directly from the copyright holder. To view a copy of this licence, visit http://creativecommons.org/licenses/by/4.0/.

\section{References}

1. S.B. Mahato, A.N. Ganguly, N.P. Sahu, Phytochemistry 21, 959$978(1982)$

2. S.L. Man, W.Y. Gao, Y.J. Zhang, L.Q. Huang, C.X. Liu, Fitoterapia 81, 703-714 (2010)

3. J.S. Kaunda, Y.J. Zhang, Nat. Product. Bioprospect. 9, 77-137 (2019)

4. J.P. Vincken, L. Heng, A. Groot, H. Gruppen, Phytochemistry 68 , 275-297 (2007)

5. M. Sautour, A.C. Mitaine-Offer, T. Miyamoto, A. Dongmo, M.A. Lacaille-Dubois, Planta Med. 70, 90-92 (2004)

6. Y. Sauvaire, G. Ribes, J.C. Baccou, M.M.L. Mariani, Lipids 26, 191-197 (1991)

7. M.J. Liu, Z. Wang, Y. Ju, J.B. Zhou, Y. Wang, R.N.S. Wong, Biol. Pharm. Bull. 27, 1059-1065 (2004)

8. T. Nikaidoa, T. Ohmotoa, S. Kubo, Y. Mimaki, Y. Sashida, Phytochemistry 31, 2445-2450 (1992)
9. I. Podolak, A. Galanty, D. Sobolewska, Phytochem. Rev. 9, 425474 (2010)

10. C. Bachran, S. Bachran, M. Sutherland, D. Bachran, H. Fuchs, Mini-Rev. Med. Chem. 8, 575-584 (2008)

11. R. Jian, K.W. Zeng, J. Li, N. Li, Y. Jiang, P.F. Tu, Fitoterapia 84, 80-84 (2013)

12. Y. Shen, C.L. Xu, W.D. Xuan, H.L. Li, R.H. Liu, X.K. Xu, H.S. Chen, Arch. Pharmacal Res. 34, 1587-1591 (2011)

13. H.J. Zhang, K. Sydara, G.T. Tan, C.Y. Ma, B. Southavong, D.D. Soejarto, J.M. Pezzuto, H.H.S. Fong, J. Nat. Prod. 67, 194-200 (2004)

14. X.N. Li, C. Chu, D.P. Cheng, S.Q. Tong, J.Z. Yan, Nat. Prod. Commun. 7, 1357-1358 (2012)

15. X.N. Li, C. Chu, D.P. Cheng, S.Q. Tong, J.Z. Yan, Chem. Nat. Compd. 50, 326-328 (2014)

16. B. Liu, B.X. Li, D. Zhou, X.Y. Wen, Y.J. Wang, G. Chen, N. Li, Bioorg. Chem. 4, 105237 (2021)

17. J.E. Sung, H.A. Lee, J.E. Kim, W.B. Yun, B.S. An, S.Y. Yang, D.S. Kim, C.Y. Lee, H.S. Lee, H.S. Lee, C.J. Bae, D.Y. Hwang, Int. J. Mol. Med. 40, 1365-1376 (2017)

18. J.E. Sung, J.Y. Choi, J.E. Kim, H.A. Lee, W.B. Yun, J.J. Park, H.R. Kim, B.R. Song, D.S. Kim, C.Y. Lee, H.S. Lee, Y. Lim, D.Y. Hwang, Lab. Anim. Res. 33, 57-67 (2017)

19. P.F. Zhu, G.G. Cheng, L.Q. Zhao, A. Khan, X.W. Yang, B.Y. Zhang, M.C. Li, Y.P. Liu, X.D. Luo, J. Agric. Food Chem. 69, 6229-6239 (2021)

20. S. Kiyosawa, M. Hutoh, T. Komori, T. Nohara, I. Hosokawa, T. Kawasaki, Chem. Pharm. Bull. 16, 1162-1164 (1968)

21. Y. Shao, O. Poobrasert, E. Kennelly, C.K. Chin, C.T. Ho, M.T. Huang, S.A. Garrison, G.A. Cordell, Planta Med. 63, 258-262 (1996)

22. P.K. Agrawal, Steroids 70, 715-724 (2005)

23. B. Shao, H.Z. Guo, Y.J. Cui, M. Ye, J. Han, D. Guo, Phytochemistry 68, 623-630 (2007)

24. J. Zhang, T.Y. Hu, G.L. Shen, Y. Yang, Z.B. Gu, Zhongchengyao 37, 2682-2686 (2015)

25. J.S. Negi, P. Singh, G.P. Joshi, M.S. Rawat, V.K. Bisht, Phcog. Rev. 4, 215-220 (2010)

26. B. Song, W.L. Huang, Y. Li, Y.Y. Zhang, H.W. Zhang, Y. Jiang, C. Deng, X.M. Song, J.L. Liu, Nat. Prod. Res. 35, 1478-6419 (2019)

27. X.J. Qin, L.J. Zhang, Y. Zhang, W. Ni, X.Z. Yang, Q. Yu, H. Yan, L.K. An, H.Y. Liu, Bioorg. Chem. 99, 103788 (2020)

28. Y. Zhang, H.Z. Li, Y.J. Zhang, M.R. Jacob, S.I. Khan, X.C. Li, C.R. Yang, Steroids 71, 712-719 (2006)

29. X.J. Qin, W. Ni, C.X. Chen, H.Y. Liu, Nat. Product. Bioprospect. 8, 265-278 (2018)

30. T.M. Buttkea, J.A. McCubreya, T.C. Owen, J. Immunol. Methods 157, 233-240 (1993)

31. U. Farooq, S. Khan, S. Naz, A. Khan, A. Khan, A. Ahmed, A. Rauf, S.M. Bukhari, S.A. Khan, A. Kamil, N. Riaz, A.R. Khan, China J. Nat. Med. 15, 944-949 (2017) 